UND-HEP-95-BIG08

July 1995

\title{
QUO VADIS, FASCINUM?
}

\author{
I.I. Bigi \\ Physics Dept., University of Notre Dame du Lac, Notre Dame, IN 46556, U.S.A. \\ e-mail address: BIGI@UNDHEP.HEP.ND.EDU
}

\begin{abstract}
The recent progress in our understanding of heavy-flavour decays allows us to define more reliably which future measurements on charm decays are needed to further advance our understanding of QCD and to get our theoretical tools ready for treating beauty decays. After sketching the theoretical landscape I list those required measurements. I argue that some - in particular those concerning inclusive semileptonic charm decays and their lepton spectra - can presumably be performed only at a tau-charm factory.
\end{abstract}

\section{Overview}

There are four questions I would like to address in reviewing the long-term goals of charm decay studies: why, how, where and when?

$$
\text { Why? }
$$

There exists a triple motivation for a detailed analysis of charm decays: (i) it provides novel probes of QCD, (ii) sharpens our tools for dealing with beauty decays and (iii) represents one of the more promising avenues to finding the hoped-for 'unexpected', namely the intervention of New Physics; this would most clearly be realized through the observation of CP asymmetries [1, 2].

\footnotetext{
${ }^{1}$ Invited Lecture given at the Workshop on the Tau/Charm Factory, Argonne National Laboratory, June 21 - 23, 1995
} 
How?

A comprehensive program is required with its cornerstones being the measurements of (a) lifetimes, (b) semileptonic branching ratios, (c) nonleptonic branching ratios and $(\mathrm{d})$ lepton spectra in exclusive as well as inclusive semileptonic charm decays.

Where?

Three different experimental environments have been considered for high-statistics charm decay studies, namely $(\alpha) B$ factories, $(\beta)$ photo- and hadro-production and $(\gamma)$ a $\tau$-charm factory.

When?

There are two benchmark dates in evaluating the impact of a $\tau$-charm factory, namely (A) 1998 - 2000: the next round of fixed target experiments at FNAL will be finished by then; the data on charm decays from CLEO will have reached a new dimension statistically and systematically; BABAR and BELLE will commence data taking;

(B) 2005: the analysis of charm and beauty decays at the $B$ factories should have reached a fully mature level; new initiatives like CHARM2000 will have had a run; the LHC with its dedicated program on beauty physics will hopefully start up.

My talk will be organized as follows: in Sect.2 I will sketch the relevant theoretical framework for charm decays; in Sect.3 I list the database that is required - or at least desired - for a deeper understanding to come about; in Sect.4 I review various experimental stages before presenting an outlook in Sect.5.

\section{Theoretical Framework}

\subsection{The Landscape}

Four second-generation theoretical technologies have emerged for treating the impact of the strong interactions on heavy-flavour decays:

- $Q C D$ Sum Rules are employed for describing exclusive semileptonic and non-leptonic decays of hadrons with strangeness, charm and beauty.

- Lattice Simulations of $Q C D$ have worked their way down in distance scale to deal with exclusive semileptonic and non-leptonic charm decays, albeit only in the quenched approximation; quite significant jumps are required before beauty decays can be tackled and one can go beyond the quenched approximation.

- Heavy Quark Effective Theory (HQET) on the other hand deals with exclusive semileptonic decays of beauty hadrons; its applicability to charm decays is dubious. 
- $1 / m_{Q}$ Expansions are distinct from the other technologies in that they deal with inclusive transitions, semileptonic as well as non-leptonic ones. Similarly to HQET they apply best to beauty decays while their numerical usefulness in charm decays is a priori uncertain.

There is one point of particular relevance for our discussion here. Charm decays occupy a special place in the theoretical landscape: they represent common ground for all the QCD methods listed above, albeit sometimes only at the extreme range of their applicability 2]. Comparing the predictions from the various theoretical technologies, which emphasize complementary aspects of QCD, with detailed and comprehensive data has a two-fold benefit:

- It provides valuable insights into the inner workings of QCD in general, and on the interplay between perturbative and non-perturbative effects in particular.

- These findings can be extrapolated to beauty decays and applied there with tested confidence.

\subsection{Inclusive Transitions}

\subsubsection{Total Rates}

Total rates for a heavy-flavour hadron $H_{Q}$ to decay into an inclusive final state $f$ can be expressed through an expansion in powers of $1 / m_{Q}$ [3]; through order $1 / m_{Q}^{3}$ one obtains the following master equation[4]:

$$
\begin{aligned}
\Gamma\left(H_{Q} \rightarrow f\right)= & \frac{G_{F}^{2} m_{Q}^{5}}{192 \pi^{3}}|K M|^{2}\left[c_{3}^{f}\left\langle H_{Q}|\bar{Q} Q| H_{Q}\right\rangle+c_{5}^{f} \frac{\left\langle H_{Q}|\bar{Q} i \sigma \cdot G Q| H_{Q}\right\rangle}{m_{Q}^{2}}+\right. \\
& \left.+\sum_{i} c_{6, i}^{f} \frac{\left\langle H_{Q}\left|\left(\bar{Q} \Gamma_{i} q\right)\left(\bar{q} \Gamma_{i} Q\right)\right| H_{Q}\right\rangle}{m_{Q}^{3}}+\mathcal{O}\left(1 / m_{Q}^{4}\right)\right]
\end{aligned}
$$

where the dimensionless coefficients $c_{i}^{f}$ depend on the parton level characteristics of $f$ (such as the ratios of the final-state quark masses to $m_{Q}$ ); $K M$ denotes the appropriate combination of KM parameters, and $\sigma \cdot G=\sigma_{\mu \nu} G_{\mu \nu}$ with $G_{\mu \nu}$ being the gluonic field strength tensor. The last term in eq.(1) implies also the summation over the four-fermion operators with different light flavours $q$.

It is through the quantities $\left\langle H_{Q}\left|O_{i}\right| H_{Q}\right\rangle$ that the dependence on the decaying hadron $H_{Q}$, and on non-perturbative forces in general, enters; they reflect the fact

\footnotetext{
${ }^{2}$ The non-perturbative contributions can be expressed through an expansion in powers of $\mu_{\text {had }} / m_{Q}$; for charm one has $\mu_{\text {had }} / m_{Q} \sim 0.4$. It is at least smaller than unity, but not by a large factor.
} 
that the weak decay of the heavy quark $Q$ does not proceed in empty space, but within a cloud of light degrees of freedom - (anti)quarks and gluons - with which $Q$ and its decay products can interact strongly. The practical usefulness of the $1 / m_{Q}$ expansion rests on our ability to determine the size of these matrix elements. Through order $1 / m_{Q}^{3}$ there are three types of expectation values that determine the non-perturbative corrections:

(i) The leading term can be expanded further:

$$
\left\langle H_{Q}|\bar{Q} Q| H_{Q}\right\rangle=1-\frac{\left\langle\left(\vec{p}_{Q}\right)^{2}\right\rangle_{H_{Q}}}{2 m_{Q}^{2}}+\frac{\left\langle\mu_{G}^{2}\right\rangle_{H_{Q}}}{2 m_{Q}^{2}}+\mathcal{O}\left(1 / m_{Q}^{3}\right)
$$

where $\left\langle\left(\vec{p}_{Q}\right)^{2}\right\rangle_{H_{Q}} \equiv\left\langle H_{Q}\left|\bar{Q}(i \vec{D})^{2} Q\right| H_{Q}\right\rangle$ denotes the average kinetic energy of the quark $Q$ moving inside the hadron $H_{Q}$ and $\left\langle\mu_{G}^{2}\right\rangle_{H_{Q}} \equiv\left\langle H_{Q}\left|\bar{Q} \frac{i}{2} \sigma \cdot G Q\right| H_{Q}\right\rangle$. The first term on the right-hand-side of eq.(2) represents the naive spectator ansatz.

(ii) The quantity $\left\langle\mu_{G}^{2}\right\rangle_{P_{Q}}$ is known from the meson hyperfine splitting:

$$
\left\langle\mu_{G}^{2}\right\rangle_{P_{Q}} \simeq \frac{3}{4}\left(M_{V_{Q}}^{2}-M_{P_{Q}}^{2}\right)
$$

where $P_{Q}$ and $V_{Q}$ denote the pseudoscalar and vector mesons, respectively. Therefore

$$
\left\langle\mu_{G}^{2}\right\rangle_{B} \simeq 0.37 \mathrm{GeV},\left\langle\mu_{G}^{2}\right\rangle_{D} \simeq 0.41 \mathrm{GeV}
$$

For $\Lambda_{Q}$ and $\Xi_{Q}$ baryons one has instead

$$
\left\langle\mu_{G}^{2}\right\rangle_{\Lambda_{Q}, \Xi_{Q}} \simeq 0
$$

since the light diquark system inside $\Lambda_{Q}$ and $\Xi_{Q}$ carries no spin, whereas

$$
\left\langle\mu_{G}^{2}\right\rangle_{\Omega_{Q}} \neq 0
$$

For $\left\langle\left(\vec{p}_{Q}\right)^{2}\right\rangle_{H_{Q}}$ there exists an estimate from a QCD sum rules analysis[5]

$$
\left\langle\left(\vec{p}_{b}\right)^{2}\right\rangle_{B} \simeq 0.5 \pm 0.1 \mathrm{GeV}
$$

and one can expect one from lattice QCD in the foreseeable future. We do have a model-independant lower bound on it [6]:

$$
\left\langle\left(\vec{p}_{b}\right)^{2}\right\rangle_{B} \geq 0.37 \pm 0.1 \mathrm{GeV} .
$$

The difference in the kinetic energy of $\mathrm{Q}$ inside baryons and mesons can be related to the masses of charm and beauty hadrons:

$$
\left\langle\left(\vec{p}_{Q}\right)^{2}\right\rangle_{\Lambda_{Q}}-\left\langle\left(\vec{p}_{Q}\right)^{2}\right\rangle_{P_{Q}} \simeq \frac{2 m_{b} m_{c}}{m_{b}-m_{c}} \cdot\left\{\left[\left\langle M_{B}\right\rangle-M_{\Lambda_{b}}\right]-\left[\left\langle M_{D}\right\rangle-M_{\Lambda_{c}}\right]\right\}
$$


where $\left\langle M_{B, D}\right\rangle$ denote the 'spin averaged' meson masses: $\left\langle M_{B}\right\rangle \equiv \frac{1}{4}\left(M_{B}+3 M_{B^{*}}\right)$ and likewise for $\left\langle M_{D}\right\rangle$. Using data one finds: $\left\langle\left(\vec{p}_{Q}\right)^{2}\right\rangle_{\Lambda_{Q}}-\left\langle\left(\vec{p}_{Q}\right)^{2}\right\rangle_{P_{Q}}=-(0.07 \pm$ $0.20)(G e V)^{2}$; i.e., the present measurement of $M_{\Lambda_{b}}$ is not yet sufficiently accurate, but this will change in the foreseeable future.

(iii) The expectation values for the four-quark operators taken between meson states can be expressed in terms of a single quantity, namely the decay constant:

$$
\left.\left\langle H_{Q}(p)\right| \bar{Q}_{L} \gamma_{\mu} q_{L}\right)\left(\bar{q}_{L} \gamma_{\nu} Q_{L}\right)\left|H_{Q}(p)\right\rangle \simeq \frac{1}{4} f_{H_{Q}}^{2} p_{\mu} p_{\nu}
$$

where factorization has been assumed. The theoretical expectations for the decay constants are [7]

$$
\begin{aligned}
& f_{D} \simeq 200 \pm 30 \mathrm{MeV} \quad, \quad f_{B} \simeq 180 \pm 30 \mathrm{MeV} \\
& f_{D_{s}} / f_{D} \simeq 1.15-1.2 \quad, \quad f_{B_{s}} / f_{B} \simeq 1.15-1.2
\end{aligned}
$$

The size of the expectation values taken between baryonic states are quite uncertain at present. There exists more than one relevant contraction, and for the time being quark model estimates provide us with the only guidance! I will return to this point when discussing predictions of baryon lifetimes.

To illustrate the method I give the semileptonic and non-leptonic widths for charm hadrons through order $1 / m_{c}^{2}$ :

$$
\begin{gathered}
\Gamma_{S L}\left(H_{c}\right) \simeq \Gamma_{0}\left\langle H_{c}|\bar{c} c| H_{c}\right\rangle \cdot\left(1-\frac{2\left\langle\mu_{G}^{2}\right\rangle_{H_{c}}}{m_{c}^{2}}+\mathcal{O}\left(1 / m_{c}^{3}\right)\right) \\
\Gamma_{N L}\left(H_{c}\right) \simeq \Gamma_{0} N_{C}\left\langle H_{c}|\bar{c} c| H_{c}\right\rangle \cdot\left[A_{0}\left(1-\frac{2\left\langle\mu_{G}^{2}\right\rangle_{H_{c}}}{m_{c}^{2}}\right)-4 A_{2} \frac{2\left\langle\mu_{G}^{2}\right\rangle_{H_{c}}}{m_{c}^{2}}+\mathcal{O}\left(1 / m_{c}^{3}\right)\right] \\
\Gamma_{0}=\frac{G_{F}^{2} m_{c}^{5}}{192 \pi^{3}}|V(c s)|^{2}
\end{gathered}
$$

where $A_{0,2}$ denote perturbative QCD corrections; I have ignored here the small phase space correction due to $m_{s}^{2} / m_{c}^{2} \neq 0$. The following results can be read off from eqs.(8):

- For $m_{c} \rightarrow \infty$ the parton model spectator expression is recovered.

- $\Gamma_{S L}$ as well as $\Gamma_{N L}$ receive non-perturbative corrections, as does $B R_{S L}\left(H_{c}\right)$; the latter quantity is lowered since the last term in eq.(8b) enhances $\Gamma_{N L}\left(A_{2}<0\right.$ !) $甘$.

- $\Gamma_{S L}$ is not universal for all charm hadrons $H_{c}$ once $\mathcal{O}\left(1 / m_{c}^{2}\right)$ contributions are included. One actually finds

$$
\Gamma_{S L}(D) / \Gamma_{S L}\left(\Lambda_{c}\right) / \Gamma_{S L}\left(\Omega_{c}\right) \sim 1 / 1.5 / 1.2
$$




\begin{tabular}{|l|l|l|}
\hline Observable & QCD $\left(1 / m_{c}\right.$ expansion) & Data \\
\hline \hline$\tau\left(D^{+}\right) / \tau\left(D^{0}\right)$ & $\begin{array}{l}\sim 2 \quad\left[\text { for } f_{D} \simeq 200 \mathrm{MeV}\right] \\
(\text { mainly due to destructive interference })\end{array}$ & $2.547 \pm 0.043$ \\
\hline$\tau\left(D_{s}\right) / \tau\left(D^{0}\right)$ & $1 \pm$ few $\times 0.01$ & $1.125 \pm 0.042$ \\
\hline$\tau\left(\Lambda_{c}\right) / \tau\left(D^{0}\right)$ & $\sim 0.5^{*}$ & $0.51 \pm 0.05$ \\
\hline$\tau\left(\Xi_{c}^{+}\right) / \tau\left(\Lambda_{c}\right)$ & $\sim 1.3^{*}$ & $1.75 \pm 0.36$ \\
\hline$\tau\left(\Xi_{c}^{+}\right) / \tau\left(\Xi_{c}^{0}\right)$ & $\sim 2.8^{*}$ & $3.57 \pm 0.91$ \\
\hline$\tau\left(\Xi_{c}^{+}\right) / \tau\left(\Omega_{c}\right)$ & $\sim 4^{*}$ & $3.9 \pm 1.7$ \\
\hline
\end{tabular}

Table 1: QCD Predictions for Charm Lifetimes

where the difference in the $\Lambda_{c}$ and $\Omega_{c}$ widths to this order are due to eqs.(4b,c); i.e., the ratio of semileptonic branching ratios does not coincide with the ratio of lifetimes when comparing mesons and baryons.

- The widths for $D$ and $\Lambda_{c}$ decays differ already in order $1 / m_{c}^{2}$.

- The differentiation between the widths for $D^{0}, D^{+}$and $D_{s}$ mesons occurs at order $1 / m_{c}^{3}$ as expressed in eq.(1), but left out in eq.(8). [

The underlying pattern can be expressed as follows:

$$
\begin{aligned}
& \Gamma\left(\Lambda_{Q}\right)=\Gamma\left(P_{Q}^{0}\right)=\Gamma\left(P_{Q}^{+}\right)+\mathcal{O}\left(1 / m_{Q}^{2}\right) \\
& \Gamma\left(\Lambda_{Q}\right)>\Gamma\left(P_{Q}^{0}\right) \simeq \Gamma\left(P_{Q}^{+}\right)+\mathcal{O}\left(1 / m_{Q}^{3}\right) \\
& \Gamma\left(\Lambda_{Q}\right)>\Gamma\left(P_{Q}^{0}\right)>\Gamma\left(P_{Q}^{+}\right)+\mathcal{O}\left(1 / m_{Q}^{4}\right)
\end{aligned}
$$

i.e., one predicts $\tau\left(\Lambda_{b}\right)<\tau\left(B_{d}\right)<\tau\left(B^{-}\right)$as well as $\tau\left(\Lambda_{c}\right)<\tau\left(D^{0}\right)<\tau\left(D^{+}\right)$. Yet keeping in mind that the $1 / m_{Q}$ expansion is at best a semi-quantitative tool for $m_{Q}=m_{c}\left(\mu_{\text {had }} / m_{c} \sim 0.4 !\right)$ one cannot expect to make precise predictions for the charm lifetime ratios. On the other hand - and that is a central point of my message - measurements of the lifetime ratios for all weakly decaying charm hadrons can be used with great profit to disentangle the various contributions in charm decays. Such an anatomy will then pave the way for more reliable predictions on beauty decays. To say it differently: a comprehensive study of charm decays can be harnessed as Nature's microscope onto the numerically smaller effects in beauty decays.

In Tables 1 and 2 I juxtapose the theoretical expectations and predictions on charm and beauty lifetime ratios [8, 9, 10, 11, 12] with present data [13, 14]. As mentioned before, at present one has to rely on quark models to estimate the size of the relevant baryonic expectation values. Thus there is a model dependance in the predictions on baryon lifetimes - in contrast to the case with meson lifetimes. This is indicated in the tables by an asterisk.

\footnotetext{
${ }^{3} S U(3)_{F l}$ symmetry is very much observed in the expectation values $\left\langle\mu_{G}^{2}\right\rangle_{D, D_{s}}$ and $\left\langle\left(\vec{p}_{c}^{2}\right)\right\rangle_{D, D_{s}}$.
} 


\begin{tabular}{|l|l|l|}
\hline Observable & QCD $\left(1 / m_{b}\right.$ expansion $)$ & Data \\
\hline \hline$\tau\left(B^{-}\right) / \tau\left(B_{d}\right)$ & $\begin{array}{l}1+0.05\left(f_{B} / 200 \mathrm{MeV}\right)^{2}[1 \pm \mathcal{O}(10 \%)]>1 \\
(\text { mainly due to destructive interference) }\end{array}$ & $1.04 \pm 0.05$ \\
\hline $\bar{\tau}\left(B_{s}\right) / \tau\left(B_{d}\right)$ & $1 \pm \mathcal{O}(0.01)$ & $0.98 \pm 0.08$ \\
\hline$\tau\left(\Lambda_{b}\right) / \tau\left(B_{d}\right)$ & $\sim 0.9^{*}$ & $0.76 \pm 0.06$ \\
\hline
\end{tabular}

Table 2: QCD Predictions for Beauty Lifetimes

The general agreement with the data is remarkable, in particular for the charm system, where the expansion parameter is not much smaller than unity. A few more detailed comments are in order:

- The $D^{+}-D^{0}$ lifetime difference is driven mainly by a destructive interference [15] with 'Weak Annihilation' (WA) contributing not more than $10-20 \%$. Within the accuracy of the expansion, the data are reproduced.

- The $D_{s}-D^{0}$ lifetime ratio can be treated with better theoretical accuracy, namely of order a few percent. The observed near equality of $\tau\left(D_{s}\right)$ and $\tau\left(D^{0}\right)$ represents rather direct evidence for the reduced weight of WA in charm meson decays [9].

- Even the expectations on the charm baryon lifetimes reproduce the data which is quite remarkable since there are constructive as well as destructive contributions to baryon lifetimes. It has to be noted though that the present measurements suffer from large uncertainties.

- However the prediction on $\tau\left(\Lambda_{b}\right) / \tau\left(B_{d}\right)$ appears to be in serious (though not yet conclusive) disagreement with the data. The details of what went into that prediction can be found in ref. [12]; here I want to state only the following conclusion. If $\tau\left(B_{d}\right)$ indeed exceeds $\tau\left(\Lambda_{b}\right)$ by 25 - $30 \%$, then a 'theoretical price' has to be paid. It strongly suggests that the present agreement between theoretical expectations and data on charm baryon lifetimes is largely accidental and most likely would not survive in the face of more precise measurements! To state it in a more constructive manner: more precise measurements on charm baryon lifetimes would then allow to isolate the source of the discrepancy between prediction and observation.

As already said, on general grounds one does not predict the semileptonic widths to be the same for all charm hadrons - apart from $\Gamma_{S L}\left(D^{0}\right) \simeq \Gamma_{S L}\left(D^{+}\right)$, which is protected by isospin invariance and Cabibbo suppression. A priori there could be a sizeable difference in $\Gamma_{S L}\left(D^{0}\right)$ vs. $\Gamma_{S L}\left(D_{s}\right)$ due to a WA contribution to the latter observable. It is then a non-trivial prediction that those two quantities largely coincide:

$$
1 \pm \sim \text { few } \%=\frac{\tau\left(D_{s}\right)}{\tau\left(D^{0}\right)} \simeq \frac{B R_{S L}\left(D_{s}\right)}{B R_{S L}\left(D^{0}\right)} \simeq 1 \pm \sim 10 \%
$$


On the other hand a sizeable difference is expected in the semileptonic widths of baryons and mesons which is expressed as follows:

$$
B R_{S L}\left(\Lambda_{c}\right)>B R_{S L}\left(D^{0}\right) \cdot \frac{\tau\left(\Lambda_{c}\right)}{\tau\left(D^{0}\right)} \simeq 0.5 \cdot B R_{S L}\left(D^{0}\right)
$$

\subsubsection{Lepton Spectra}

A detailed study of the lepton spectra in inclusive semileptonic decays of $D^{0}, D^{+}$ and $D_{s}$ mesons is highly desirable. One expects [16 sizeable differences between the energy spectra in $D^{0}$ and $D_{s}$ and to a lesser degree also in $D^{+}$decays. For there is a WA process that is Cabibbo allowed [forbidden] for $D_{s}\left[D^{+}\right]$mesons where the hadrons in the final state emerge from (double) gluon emission of the initial anti-quark line. These differences will show up mainly in the endpoint region. An analogous complication is expected for semileptonic $B$ decays: hadronization affects the spectra in the endpoint region differently in $B_{d}$ and $B^{-}$transitions. This creates a systematic uncertainty in the value extracted from inclusive decays that cannot be evaluated reliably unless

- one separates $B_{d}$ and $B^{-}$decays or

- measures the corresponding effects for $D$ mesons and extrapolates to $B$ mesons through a $1 / m_{Q}$ expansion.

\subsection{Exclusive Charm Decays}

\subsubsection{Leptonic and Semileptonic Channels}

Measuring $B R\left(D^{+}, D_{s} \rightarrow \mu \nu, \tau \nu\right)$ with good accuracy represents a high priority goal since it allows to extract the decay constants $f_{D}$ and $f_{D_{s}}$. There exists considerable intrinsic interest in the value of these hadronic parameters; in addition - and maybe more urgently from a phenomenological perspective - once $f_{D}$ and $f_{D_{s}}$ have been well measured, one can confidently extrapolate to the beauty sector and predict $f_{B}$ and $f_{B_{s}}$.

As discussed in detail in El'Khadra's talk [17] a host of theoretical tools can be brought to bear on exclusive semileptonic charm decays: QCD sum rules, Lattice QCD and HQET in addition to quark models. Confronting their predictions with comprehensive measurements of the relevant hadronic form factors and their dependance on the momentum transfer will provide us with valuable insights into the inner workings of QCD; it also will be of great benefit in quantitatively understanding exclusive semileptonic $B$ decays. 


\subsubsection{Nonleptonic Modes}

Most important is a general caveat: the relationship between the pattern in exclusive modes and in inclusive transitions is quite tenuous. The former in contrast to the latter are very sensitive to the dramatic behaviour of QCD in the infrared regime; there exist relatively straightforward examples [8] showing that while individual exclusive rates get enhanced or decreased significantly by the strong interactions, these effects average out to a large degree in the sum. No theoretical tools have been developed yet that can master these complexities and at present one can employ only models of uncertain reliability. Nevertheless there is a valid motivation behind such 'phenomenological engineering', in particular when applied to two-body modes. For it allows us - once sufficiently many branching ratios have been well measured - to extract the size of the contributing isospin amplitudes and their phase shifts [18]. This provides information that is essential for designing a strategy for CP studies and for interpreting its outcome.

\subsubsection{Radiative Decays}

While in the Standard Model there is no (short-distance) penguin operator generating $D \rightarrow \gamma+X, \gamma K^{*}, \gamma \rho / \omega$ transitions, long distance dynamics can. One should note that even the inclusive rate receives contributions from a non-local (though higherdimensional) operator. Thus the radiative branching ratios cannot be predicted in a reliable fashion. Yet measuring $B R\left(D \rightarrow \gamma K^{*}, \gamma \rho / \omega\right)$ helps us in two ways: On the one hand one can again extrapolate to the beauty system and obtain a reliable estimate for the impact of long-distance dynamics on the corresponding modes $B \rightarrow$ $\gamma K^{*}, \gamma \rho / \omega$. This is important for any attempt to extract $|V(u b)|$ from these radiative $B$ decays. On the other hand one has opened up a new window onto New Physics; for it can manifest itself by producing a significant deviation of the ratio $B R(D \rightarrow$ $\gamma \rho / \omega) / B R\left(D \rightarrow \gamma K^{*}\right)$ from $\tan ^{2}\left(\theta_{c}\right)$.

\section{Required/Desired Database}

The preceding discussion should have made it clear that even without aiming at possible manifestations of New Physics the need for further data on charm decays has not diminished; the advances in our theoretical understanding actually allow us to define more precisely the kind of future measurement one needs for further progress. I will briefly sketch them.

While there is no need from theory to measure the $D^{+}, D^{0}$ and $\Lambda_{c}$ lifetimes more

precisely, it would be quite useful to determine $\tau\left(D_{s}\right)$ to within $1 \%$. Clearly the 
greatest need for improvement exists for the $\Xi_{c}$ and $\Omega_{c}$ lifetimes. A 5-10\% accuracy in $\tau\left(\Xi_{c}^{0}\right), \tau\left(\Xi_{c}^{+}\right)$and $\tau\left(\Omega_{c}\right)$ would enable us to extract the size of the relevant baryonic matrix elements.

The benchmark to aim for in $B R\left(D^{+}, D_{s} \rightarrow \mu \nu, \tau \nu\right)$ is a $10 \%$ accuracy allowing to extract the decay constants to within $5 \%$.

For practical reasons one wants to know the absolute branching ratios for charm hadrons to within a few percent. Such information which is sorely missing for $D_{s}$, $\Lambda_{c}$ and $\Xi_{c}$ decays $19 \pi$ is needed, among other things, for proper charm counting in $B$ decays and for determining the absolute values of $B R\left(B_{s} \rightarrow l \nu D_{s}^{(*)}\right), B R(B \rightarrow$ $\left.D \bar{D}_{s}^{(*)}\right)$ and $B R\left(B_{s} \rightarrow D_{s}^{(*)} \bar{D}_{s}^{(*)}\right)$. Likewise one wants to know the inclusive rates for $\Lambda_{c} \rightarrow \Xi+X_{s}, \Xi_{c} \rightarrow \Xi+X$ etc. Our ignorance here constitutes a major bottle neck in many studies, like using $l \Xi$ correlations to distinguish between $\Lambda_{b}$ and $\Xi_{b}$ decays.

It is also important to know the absolute branching ratios for the inclusive transitions $D_{s} \rightarrow l+X, \Lambda_{c} \rightarrow l+X, \Xi_{c} \rightarrow l+X$ to complement the information obtained from the lifetimes. A detailed analysis of the lepton spectra in $D, D_{s} \rightarrow l+X$ (and also in $\left.\Lambda_{c} \rightarrow l+X\right)$ would be of great theoretical help when extracting $|V(u b)|$ from the endpoint region in $B \rightarrow l+X$ decays.

The dependance of the hadronic form factors in exclusive semileptonic charm decays on the momentum transfer has to be measured directly and the analysis has to be extended to include also channels like $D^{+}, D_{s} \rightarrow l \nu \eta / \eta^{\prime}$.

The data base for the program of 'theoretical engineering' in two-body modes referred to above has to be completed by analysing final states containing (multi)neutrals.

Enough statistics has to be accumulated to study doubly Cabibbo suppressed decays in detail.

The radiative channels $D, D_{s} \rightarrow \gamma K^{*}, \gamma \rho / \omega$ have to be searched for in a dedicated manner.

Finally it would be quite useful to remeasure the reaction $e^{+} e^{-} \rightarrow D \bar{D}+X$ for $E_{c . m .} \sim 5-6 \mathrm{GeV}$. Old SPEAR data suggest an enhancement there; if true, it would

point to rather virulent final state interactions in that interval. That region happpens to be the one that is also probed in $B \rightarrow D \bar{D}_{(s)}$; such effects would have an obvious impact on the $\mathrm{CP}$ phenomenology in those $B$ decays.

\section{Experimental Stage}

The measurements listed above fall into three categories:

(A) The lifetimes can be measured by fixed target experiments (or at $B$ factories). 
(B) Some of the measurements might not be impossible in hadronic collisions or at a $B$ factory, but certainly represent a stiff challenge there [20]. Determining $B R\left(D^{+}, D_{s} \rightarrow \mu \nu\right)$ with a $20 \%$ accuracy presumably belongs into that category, as do observing non-leptonic decays with multi-neutrals in the final state and extracting absolute branching ratios for $D_{s}$ mesons and the charmed baryons. A $\tau$-charm factory on the other hand offers the cleanest measurements [19].

(C) There are finally measurements that presumably will remain in the sole domain of a $\tau$-charm factory. Among them are: studies of the lepton spectra in $D / D_{s} / \Lambda_{c} \rightarrow l+X$; the semileptonic branching ratios for the various charm hadrons and the identification of genuine radiative $D$ decays which requires the efficient rejection of nonleptonic modes like $D \rightarrow K^{*} \pi^{0} \rightarrow K^{*} \gamma[\gamma]$, i.e. where one photon escapes detection; this can probably be achieved only by making use of the excellent energy resolution available due to beam-energy constraints at a threshold machine.

\section{Outlook}

Let me start out with some very general statements which I then relate to the purpose of our meeting. A comprehensive program on Heavy Flavour Physics is essential in any serious quest to unveil Nature's Grand Design. Detailed studies of charm decays have to form an integral part of such a program. In an 'ideal' or at least 'optimal' world a $\tau$-charm factory plays a central role in such studies. This factory is justified by its unique capabilities to advance our understanding of QCD, and it would run with good luminosity in the energy range $3 \mathrm{GeV} \leq \sqrt{s} \leq 6 \mathrm{GeV}$; its discovery potential for New Physics would serve as the 'icing on the cake'. The 'ideal' world is defined as one where a $\tau$-charm factory would be running by now; in an 'optimal' world it would start delivering data by the end of the millenium, like the asymmetric $B$ factories. Not surprisingly, our world is not ideal and probably not optimal. There is still an excellent motivation for a first-class $\tau$-charm factory starting up later, yet the emphasize will shift somewhat. High precision studies of $\tau$ and charmonium physics would represent the superb primary justification. On the other hand, the battle lines for open charm physics might be re-drawn. I expect that our experimental colleagues will devise some ingenious new methods for obtaining at least decent measurements of absolute branching ratios for charmed baryons etc. For cost and time (also running time) reasons it might make more sense then to limit the effective energy range of the machine to $3 \mathrm{GeV} \leq \sqrt{s} \leq 4.4 \mathrm{GeV}$ and concentrate on the core part of open charm physics, namely the weak decays of $D$ and $D_{s}$ mesons with a two-fold purpose: to perform measurements of absolute branching ratios, semileptonic decays and their lepton spectra that cannot be made in other experimental environments - and to follow up on tantalizing hints for the intervention of New Physics that might have surfaced in the meantime! 
Acknowledgements: I am grateful to T.D. Lee for sharing his insights and his enthusiasm with us. It was a stimulating meeting nicely organized by J. Repond. This work was supported by the National Science Foundation under grant number PHY 92-13313. I also thank the Institute for Nuclear Theory at the University of Washington for its hospitality and the Department of Energy for partial support during the write-up of this manuscript.

\section{References}

[1] G. Burdman, these Proceedings.

[2] I.I. Bigi, Invited talk given at HQ94, Charlottesville, Virginia, Oct. 1994, preprint UND-HEP-94-BIG11, to appear in the Proceedings.

[3] for the first suggestion, see: M. Shifman, M. Voloshin, 1982, in: V. Khoze, M. Shifman, Uspekhi Fiz. Nauk 140 (1983) 3 [Sov. Phys. Uspekhi (1983) 387]; Sov. Journ. Nucl. Phys. 41 (1985) 120.

[4] I.I. Bigi, N.G. Uraltsev, A. Vainshtein, Phys. Lett. B293 (1992) 430; (E) B297 (1993) 477; B. Blok, M. Shifman, Nucl. Phys. B399 (1993) 441; 459.

[5] P. Ball, V. Braun, Phys. Rev. D49 (1994) 2472.

[6] I.I. Bigi, M. Shifman, N.G. Uraltsev, A. Vainshtein, Phys. Rev. D52 (1995) 196, with references to earlier work.

[7] G. Martinelli, Invited talk given at the 6th Intern. Symposium on Heavy Flavour Physics, Pisa, Italy, June 1995, to appear in the Proceedings.

[8] I.I. Bigi, N.G. Uraltsev, Phys. Lett. B280 (1992) 120; B. Blok, M. Shifman, in: Proceedings of the Third Workshop on the Physics at a Tau-Charm Factory, Marbella, Spain, June 1993, R. \& J. Kirkby (eds.), Editions Frontieres, 1994.

[9] I.I. Bigi, N.G. Uraltsev, Z. Physik C 62 (1994) 623.

[10] I. I. Bigi, B. Blok, M. Shifman, N. Uraltsev, A. Vainshtein, in: 'B Decays', ed. by S. Stone, World Scientific, Revised Second Edition, 1994, p.132.

[11] note also the earlier predictions: N. Bilic, B. Guberina, J. Trampetic, Nucl. Phys. B248 (1984) 261; M. Shifman, M. Voloshin, Sov. J. Nucl. Phys. 41 (1985) 120; JETP64 (1986) 698; B. Guberina, R. Rückl, J. Trampetic, Z. Phys. C33 (1986) 297; R. Rückl,

in: Proc. International School of Physics "Enrico Fermi", Course XCII, Varenna, 1984, ed. by N. Cabibbo, North-Holland, Amsterdam, 1987, p. 43. 
[12] for an update, see: I. Bigi, Invited talk given at the 6th Intern. Symposium on Heavy Flavour Physics, Pisa, Italy, June 1995, preprint UND-HEP-95-BIG06, to appear in the Proceedings.

[13] S. Malvezzi, Invited talk given at the 6th Intern. Symposium on Heavy Flavour Physics, Pisa, Italy, June 1995, to appear in the Proceedings.

[14] V. Sharma, Invited talk given at the 6th Intern. Symposium on Heavy Flavour Physics, Pisa, Italy, June 1995, to appear in the Proceedings.

[15] B. Guberina, S. Nussinov, R. Peccei, R. Rückl, Phys. Lett. B89 (1979) 111.

[16] I.I. Bigi, N.G. Uraltsev, Nucl. Phys. B423 (1994) 33.

[17] A. El'Khadra, these Proceedings.

[18] For a recent analysis, see: F. Bucella et al., Phys. Lett. B302 (1993) 319.

[19] P. Roudeau, these Proceedings.

[20] J. Wiss, these Proceedings. 